\title{
Dynamics and Complexity Analysis of Fractional-Order Chaotic Systems with Line Equilibrium Based on Adomian Decomposition
}

\author{
Heng Chen, ${ }^{1}$ Tengfei Lei $\mathbb{D}^{2,3}$ Su Lu, ${ }^{4}$ WenPeng Dai, ${ }^{2}$ Lijun Qiu, ${ }^{1}$ and Lin $\mathrm{Zhong}^{1}$ \\ ${ }^{1}$ Shaanxi Engineering Research Center of Controllable Neutron Source, School of Science, Xijing University, Xi'an 710123, China \\ ${ }^{2}$ Collaborative Innovation Center of Memristive Computing Application, Qilu Institute of Technology, Jinan 250200, \\ Shandong, China \\ ${ }^{3}$ Shandong Engineering Research Center of China-Germany Smart Factory Applied, Jinan 250200, Shandong, China \\ ${ }^{4}$ School of Civil Engineering, Inner Mongolia University of Technology, Inner Mongolia, Huhehot 010051, China \\ Correspondence should be addressed to Tengfei Lei; leitengfeicanhe@126.com
}

Received 20 April 2020; Revised 25 May 2020; Accepted 4 June 2020; Published 22 October 2020

Guest Editor: Shao-Bo He

Copyright $\odot 2020$ Heng Chen et al. This is an open access article distributed under the Creative Commons Attribution License, which permits unrestricted use, distribution, and reproduction in any medium, provided the original work is properly cited.

\begin{abstract}
In this paper, the Adomian decomposition method (ADM) is applied to solve the fractional-order system with line equilibrium. The dynamics of the system is analyzed by means of the Lyapunov exponent spectrum, bifurcations, chaotic attractor, and largest Lyapunov exponent diagram. At the same time, through the Lyapunov exponent spectrum and bifurcation graph of the system under the change of the initial value, the influence of fractional order $q$ on the system state can be observed. That is, integer-order systems do not have the phenomenon of attractors coexistence, while fractional-order systems have it.
\end{abstract}

\section{Introduction}

Three hundred years ago, fractional-order calculation was proposed as a classical mathematical problem. Fractional calculation has no practical background and has not been applied in engineering. Therefore, researchers and scientists are not interested in fractional research. Recently, it have been found that many engineering and physical systems, electronic systems, etc. exhibit their own fractional-order characteristics, and the fractional order can more accurately reflect natural phenomena, such as material memory and damping characteristics [1-4]. In recent years, with the deepening and perfection of chaotic theory research, fractional-order chaotic systems have become a hot topic in the field. In particular, the complexity of fractional-order chaotic systems is not only related to the parameters of the system itself, but also related to the fractional order of the system. Many scholars have proposed several fractionalorder chaotic systems based on integer-order chaotic systems: fractional-order Lorenz system [5], factional-order
Lorenz hyperchaotic system [6], four-wing fractional-order chaotic system [7], and so on [8,9].

In the numerical calculation of fractional chaotic systems, namely, discretization of fractional chaotic systems, many scholars have made some achievements based on frequency-domain method (FDM) [10], Adomian decomposition method (ADM) [5-7, 9, 11], and Adams-Bashforth-Moulton (ABM) algorithm [12, 13]. Among them, FDM uses high-dimensional system close to fractional-order system through Laplace change, but the error is relatively large [14]. ABM is the most commonly used method, but its operation speed is slow. ADM scheme, on the other hand, has more accuracy and requires less computational resources compared with $\mathrm{ABM}$ algorithm $[5-7,9]$. Reference [15] shows that fractional-order chaotic systems show more complex chaotic behaviors than integerorder chaotic systems, which can be seen from complexity, Lyapunov index, bifurcation diagram, and other aspects. When the system order is smaller, the system is more complicated $[9,16]$. 
On the other hand, hot research on chaotic systems mainly focuses on chaos control [17-20], Lyapunov exponent calculation and analysis [21,22], and doubling and growth of attractors [23, 24], and chaotic systems with hidden attractors are also hot research topics, because such systems are extremely prone to multistable phenomenon, which is a common phenomenon in nature. Hidden attractors originate from several types of dynamical systems; the dynamical systems with one stable equilibrium [25], a line or plane equilibrium [26,27], or no equilibrium [28] can all have hidden attractors. Hidden attractors are even found in some special chaotic systems, which have both unstable and stable equilibria. However, the fractional-order chaotic system with hidden attractor, which can be easily obtained by combining the hidden attractor with fractional-order systems, is rarely studied [29]. The system has high complexity, so it plays an important role in the field of communication encryption.

In this paper, we propose a new fractional-order chaotic system with line equilibrium. Discretization of the proposed system is performed using Adomian decomposition scheme. The rest of the paper is organized as follows. In Section 2, the equilibrium point and dynamic analysis of the integer-order chaotic equilibrium point system are presented. In Section 3, the fractional-order chaotic system is numerically calculated and simulated by Adomian decomposition method. In Section 4, the bifurcation diagram, phase diagrams, and Lyapunov exponents spectrum are employed to analyze the dynamics of the system. In Section 5, complexity of fractional-order chaotic system is analyzed. Finally, the obtained results are summarized in Section 6.

\section{Integer-Order Chaotic System with Line Equilibrium}

2.1. Analysis of Equilibrium Points of Systems. Reference [29] proposes a class of $T$ chaotic systems defined by

$$
\left\{\begin{array}{l}
\dot{x}=a(y-x), \\
\dot{y}=c x-a x z, \\
\dot{z}=x y-b z .
\end{array}\right.
$$

A $3 \mathrm{D}$ integer-order chaotic system is defined by

$$
\left\{\begin{array}{l}
\dot{x}=y z-a x, \\
\dot{y}=c x-a x z, \\
\dot{z}=x y-b z,
\end{array}\right.
$$

where, $a, b$, and $c$ are system (2) parameters and $x, y$, and $z$ are three state variables of system (2). When parameters $a=5, b=2$, and $c=34$, the system has two equilibrium points: $E_{1}\left((c \sqrt{a b}) / a^{2}, \sqrt{a b},(c / a)\right), E_{2}\left(-\left(c \sqrt{a b} / a^{2}\right),-\sqrt{a b}\right.$, $(c / a))$, and $E_{3}(0, h, 0)$. The eigenvalues at $E_{1}$ and $E_{2}$ are $\lambda_{11}=-6.0739, \quad \lambda_{12}=-0.4631+21.3673, \quad$ and $\quad \lambda_{13}=0$; $E_{3}(0, h, 0)$ is the line equilibrium point; and $h$ is any real number. The Jacobian matrix linearized under equilibrium $E_{3}$ is as follows:

$$
J=\left[\begin{array}{ccc}
-a & 0 & h \\
c & 0 & 0 \\
h & 0 & -b
\end{array}\right] .
$$

The characteristic equation of matrix is

$$
\lambda^{3}+(a+b) \lambda^{2}+\left(\mathrm{ab}-h^{2}\right) \lambda=0
$$

The eigenvalue at the line equilibrium point $E_{3}$ is as follows:

$$
\begin{aligned}
& \lambda_{1}=0, \\
& \lambda_{2}=-\frac{(a+b)}{2}-\frac{\sqrt{(a-b)^{2}+4 h^{2}}}{2}, \\
& \lambda_{3}=-\frac{(a+b)}{2}+\frac{\sqrt{(a-b)^{2}+4 h^{2}}}{2} .
\end{aligned}
$$

Case 1. If $h^{2}<a b, \quad$ then $\lambda_{3}=-((a+b) / 2)+\left(\sqrt{(a-b)^{2}+4 h^{2}} / 2\right)<0 ;$ according to Routh-Hurwitz criterion, the equilibrium $E_{3}$ is stable.

Case 2. If $\quad h^{2}<a b, \quad$ then $\lambda_{3}=-((a+b) / 2)+\left(\sqrt{(a-b)^{2}+4 h^{2}} / 2\right)>0 ;$ according to Routh-Hurwitz criterion, the equilibrium $E_{3}$ is unstable.

Case

$$
\text { 3. If } h^{2}=a b \text {, }
$$

then

$\lambda_{3}=-((a+b) / 2)+\left(\sqrt{(a-b)^{2}+4 h^{2}} / 2\right)=0 ;$ according to Routh-Hurwitz criterion, we obtain that the equilibrium $E_{3}$ is critical stable.

When $a=5, b=2$, and $c=34$, using ODE45 function in MATLAB software to simulate (2), the chaotic attractor is shown in Figure 1. The orbits marked by the blue and red colors emerge form initial $(1,2,3)$ and $(1,2,-1)$, and the red attractor is periodic. The blue attractors are shown in chaotic states.

2.2. Dynamics Analysis of the Chaotic System. Fix $a=5, b=2$, and $c=34$; initial values of state variables $\left[x_{0}, y_{0}, z_{0}\right]=[1$, $2, z_{0}$ ]; let the $z_{0}$ vary from -15 to 15 with step size of 0.01 . Bifurcation diagram and Lyapunov exponents spectrum of system (2) are shown in Figure 2. As can be seen from the bifurcation diagram of the system in Figure 2(a), when $z_{0} \in[-15,-0.6]$, the system is in a periodic state and 


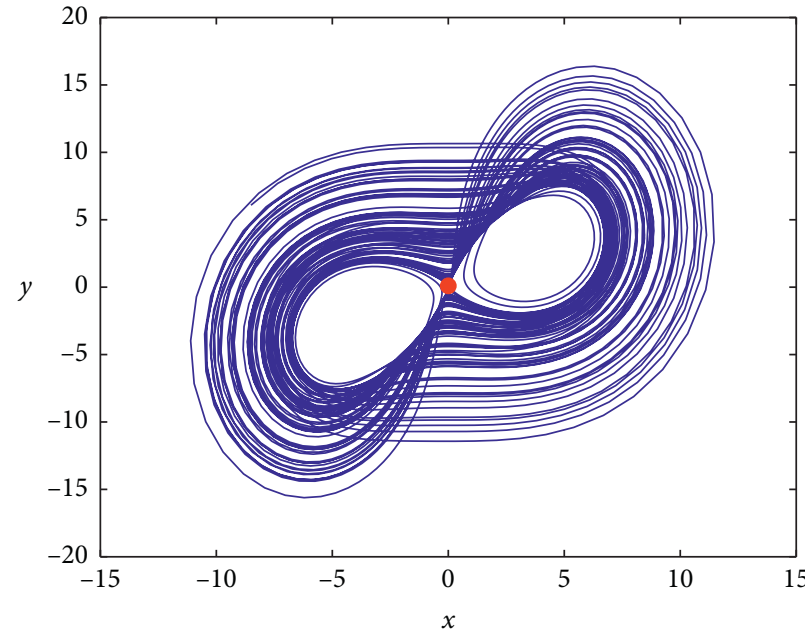

(a)

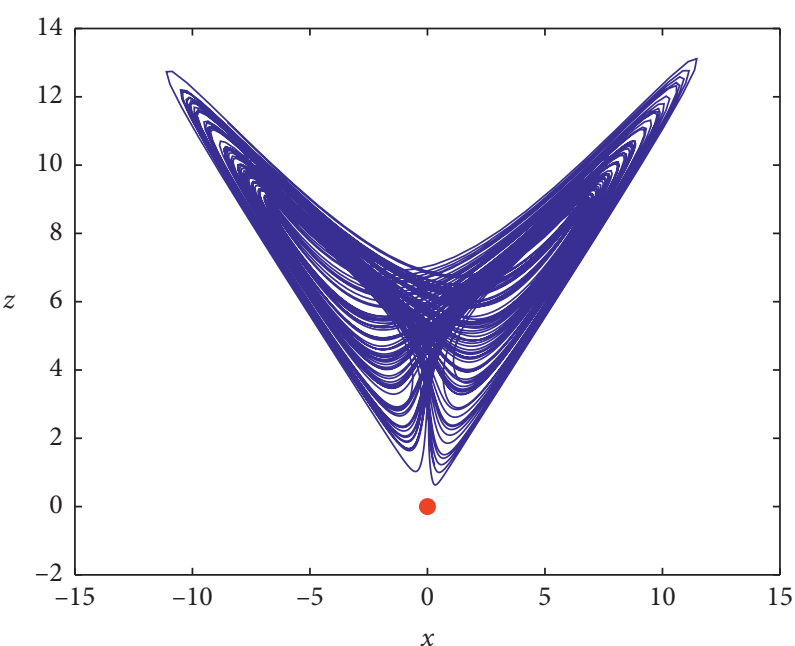

(b)

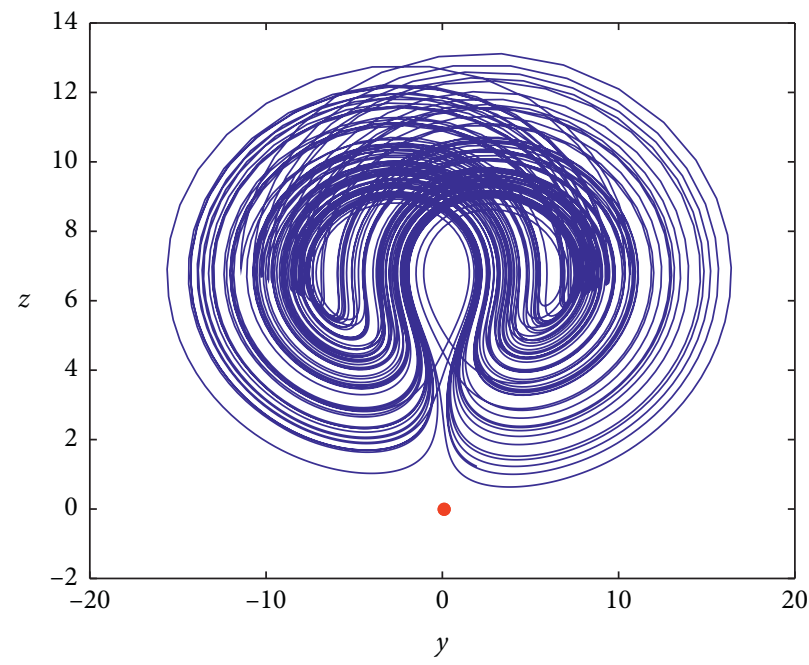

(c)

Figure 1: Phase diagrams in different projections of chaotic systems (2) from initial values $(1,2,3)$, red, and $(1,2,-1)$. (a) Phase portraits in the $x-y$ plane. (b) Phase portraits in the $x-z$ plane. (c) Phase portraits in the $y-z$ plane.

stable at $(0,0,0)$. When $z_{0} \in(-0.6,15]$, the system is in a chaos state. When $z_{0} \in[-15,-0.6]$, the maximum Lyapunov exponent is equal to 0 . When $z_{0} \in(-0.6,15]$, the largest Lyapunov exponent is positive, as shown in Figure 2(b).

Fix $a=5, b=2$, and $c=34$; initial values of state variables $\left[x_{0}, y_{0}, z_{0}\right]=\left[1,2, z_{0}\right] ;$ let the $z(0)$ vary from -15 to 15 with step size of 0.3 and $y(0)$ vary from -20 to 20 with step size of 0.4. In the $z(0)-y(0)$ plane, the chaos diagram of the largest LEs is calculated and shown in Figure 3. In Figure 3 the chaotic region is green (labeled "C") and the periodic region is red (labeled "P"). We can see from Figure 3 that the system state is mainly affected by the initial condition $z_{0}$.

\section{Solution of Fractional Chaotic System Based on Adomian Decomposition}

System (2) can generate complex chaotic attractors with line equilibrium point, and it has three typical parameter sets. Change the above system equations to the following fractional-order form: 


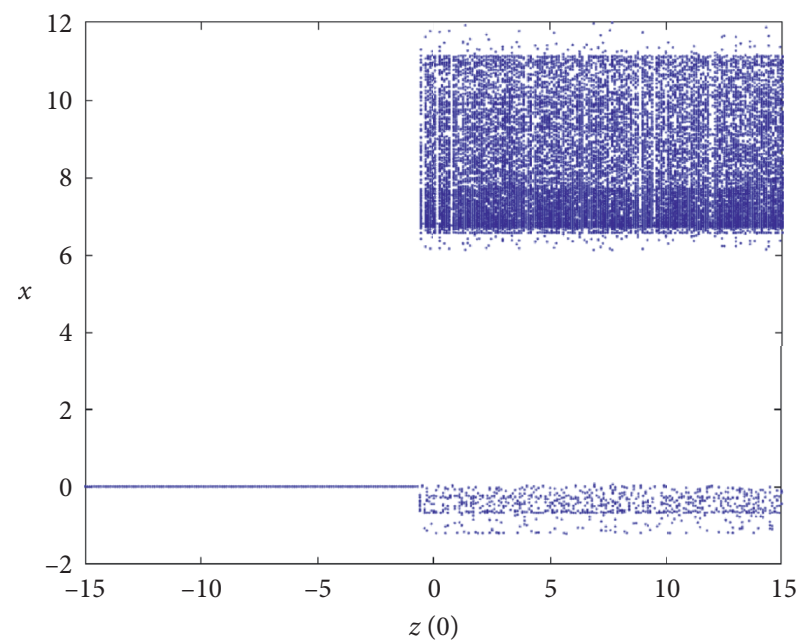

(a)

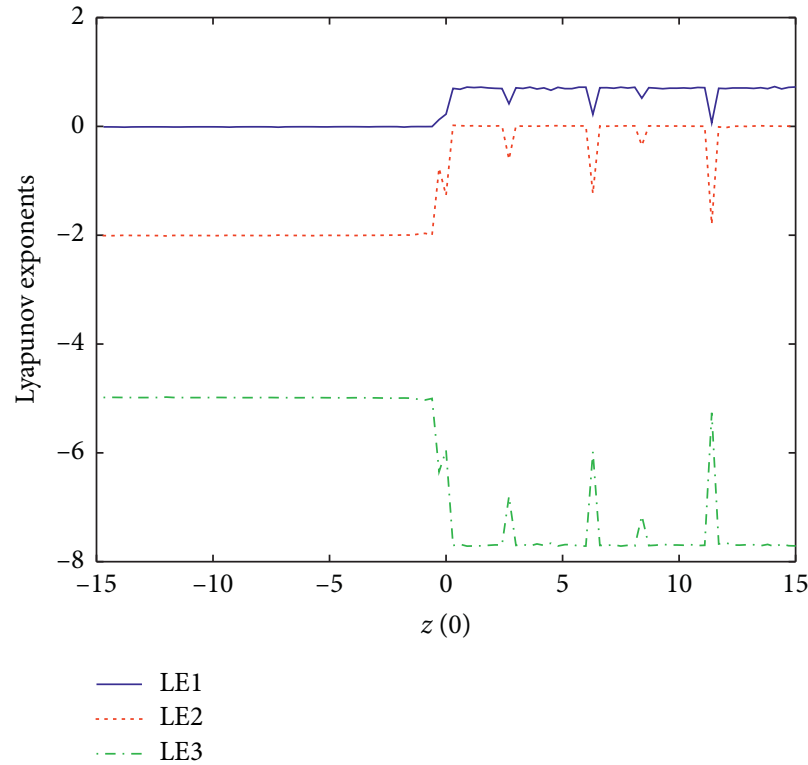

(b)

Figure 2: Bifurcation diagram and Lyapunov exponents spectrum of system (2) with initial conditional $\left[x_{0}, y_{0}, z_{0}\right]=\left[1,2, z_{0}\right]$. (a) Bifurcation diagram. (b) Lyapunov exponents (LEs).

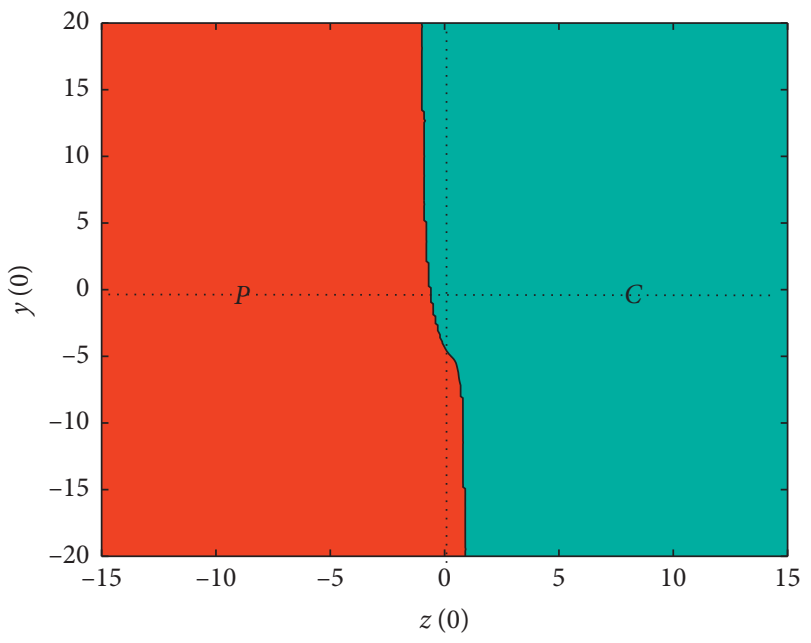

FIgURE 3: The chaos diagram of the largest Lyapunov exponents with $z_{0} \in[-15,15]$ and $y_{0} \in[-20,20]$.

$\left\{\begin{array}{l}\frac{\mathrm{d} x^{q}}{\mathrm{~d}^{q} t}=y z-\mathrm{a} x, \\ \frac{\mathrm{d} y^{q}}{\mathrm{~d}^{q} t}=\mathrm{c} x-a \mathrm{x} z, \\ \frac{\mathrm{d} z^{q}}{\mathrm{~d}^{q} t}=\mathrm{x} y-\mathrm{bz} . \\ \text { The initial condition is }\left\{\begin{array}{l}x^{0}=x\left(t_{0}\right)=c_{1}^{0} \\ y^{0}=y\left(t_{0}\right)=c_{2}^{0} \\ z^{0}=z\left(t_{0}\right)=c_{3}^{0}\end{array} . \text { According to }\right.\end{array}\right.$ domains decomposition method [30] and fractional calculus properties, the following properties are obtained:

$$
\left\{\begin{array}{l}
x^{1}=\left(c_{2}^{0} c_{3}^{0}-a c_{1}^{0}\right) \frac{h^{q}}{\Gamma(q+1)} \\
y^{1}=\left[c c_{1}^{0}-a c_{1}^{0} c_{3}^{0}\right] \frac{h^{q}}{\Gamma(q+1)} \\
z^{1}=\left(c_{1}^{0} c_{2}^{0}-b c_{3}^{0}\right) \frac{h^{q}}{\Gamma(q+1)}
\end{array}\right.
$$

where $x^{1}, y^{1}, z^{1}$ are the values of systems (3). $h_{s}=t-t_{0}$ is iteration step size. $\Gamma(\cdot)$ is Gamma function. The corresponding variables are assigned to the corresponding value; let 


$$
\begin{aligned}
& \left\{\begin{array}{l}
c_{1}^{1}=c_{2}^{0} c_{3}^{0}-a c_{1}^{0}, \\
c_{2}^{1}=c c_{1}^{0}-a c_{1}^{0} c_{3}^{0}, \\
c_{3}^{1}=c_{1}^{0} c_{2}^{0}-b c_{3}^{0},
\end{array}\right. \\
& \left\{\begin{array}{l}
c_{1}^{2}=c_{2}^{0} c_{3}^{1}+c_{2}^{1} c_{3}^{0}-a c_{1}^{1}, \\
c_{2}^{2}=c c_{2}^{1}-a\left(c_{1}^{1} c_{3}^{0}+c_{1}^{0} c_{3}^{1}\right), \\
c_{3}^{2}=c_{1}^{1} c_{2}^{0}+c_{1}^{0} c_{2}^{1}-b c_{3}^{1},
\end{array}\right. \\
& \left\{\begin{array}{l}
c_{1}^{3}=c_{2}^{2} c_{3}^{0}+c_{2}^{0} c_{3}^{2}+c_{2}^{1} c_{3}^{1} \frac{\Gamma(2 q+1)}{\Gamma^{2}(q+1)}-a c_{1}^{2}, \\
c_{2}^{3}=c c_{1}^{2}-a c_{1}^{2} c_{3}^{0}-a c_{1}^{0} c_{3}^{2}-a c_{1}^{1} c_{3}^{1} \frac{\Gamma(2 q+1)}{\Gamma^{2}(q+1)} \\
c_{3}^{3}=c_{1}^{2} c_{2}^{0}+c_{1}^{0} c_{2}^{2}+c_{1}^{1} c_{2}^{1} \frac{\Gamma(2 q+1)}{\Gamma^{2}(q+1)}-b c_{3}^{2}
\end{array}\right. \\
& \left\{\begin{array}{l}
\left.c_{1}^{4}=c_{2}^{3} c_{3}^{0}+c_{2}^{0} c_{3}^{3}+\left(c_{2}^{1} c_{3}^{2}+c_{2}^{2} c_{3}^{1}\right) \frac{\Gamma(3 q+1)}{\Gamma(2 q+1) \Gamma(q+1)}-a c_{1}^{3}\right), \\
c_{2}^{4}=c c_{1}^{3}-a c_{1}^{3} c_{3}^{0}-a c_{1}^{0} c_{3}^{3}-a\left(c_{1}^{1} c_{3}^{2}+c_{1}^{2} c_{3}^{1}\right) \frac{\Gamma(3 q+1)}{\Gamma(2 q+1) \Gamma(q+1)}, \\
c_{3}^{4}=c_{1}^{3} c_{2}^{0}+c_{1}^{0} c_{2}^{3}+\left(c_{1}^{1} c_{2}^{2}+c_{1}^{2} c_{2}^{1}\right) \frac{\Gamma(3 q+1)}{\Gamma(2 q+1) \Gamma(q+1)}-b c_{3}^{3},
\end{array}\right. \\
& \left\{\begin{array}{l}
c_{1}^{5}=c_{2}^{4} c_{3}^{0}+c_{2}^{0} c_{3}^{4}+\left(c_{2}^{1} c_{3}^{3}+c_{2}^{3} c_{3}^{1}\right) \frac{\Gamma(4 q+1)}{\Gamma(3 q+1) \Gamma(q+1)}+c_{2}^{2} c_{3}^{2} \frac{\Gamma(4 q+1)}{\Gamma^{2}(2 q+1)}-a c_{1}^{4}, \\
c_{2}^{5}=c c_{1}^{4}-a c_{1}^{4} c_{3}^{0}-a c_{1}^{0} c_{3}^{4}-a\left(c_{1}^{1} c_{3}^{3}+c_{1}^{3} c_{3}^{1}\right) \frac{\Gamma(4 q+1)}{\Gamma(3 q+1) \Gamma(q+1)}-a c_{1}^{2} c_{3}^{2} \frac{\Gamma(4 q+1)}{\Gamma^{2}(2 q+1)} \\
c_{3}^{5}=c_{1}^{4} c_{2}^{0}+c_{1}^{0} c_{2}^{4}+\left(c_{1}^{1} c_{2}^{3}+c_{1}^{3} c_{2}^{1}\right) \frac{\Gamma(4 q+1)}{\Gamma(3 q+1) \Gamma(q+1)}+c_{1}^{2} c_{2}^{2} \frac{\Gamma(4 q+1)}{\Gamma^{2}(2 q+1)}-b c_{3}^{4},
\end{array}\right. \\
& \left\{\begin{array}{l}
c_{1}^{6}=c_{2}^{5} c_{3}^{0}+c_{2}^{0} c_{3}^{5}+\left(c_{2}^{2} c_{3}^{3}+c_{2}^{3} c_{3}^{3}\right) \frac{\Gamma(5 q+1)}{\Gamma(3 q+1) \Gamma(2 q+1)}+\left(c_{2}^{1} c_{3}^{4}+c_{2}^{4} c_{3}^{1}\right) \frac{\Gamma(5 q+1)}{\Gamma(4 q+1) \Gamma(q+1)}-a c_{1}^{5}, \\
c_{2}^{6}=c c_{1}^{5}-a c_{1}^{5} c_{3}^{0}-a c_{1}^{0} c_{3}^{5}-a\left(c_{1}^{2} c_{3}^{3}+c_{1}^{3} c_{3}^{2}\right) \frac{\Gamma(5 q+1)}{\Gamma(3 q+1) \Gamma(2 q+1)}-a\left(c_{1}^{1} c_{3}^{4}+c_{1}^{4} c_{3}^{1}\right) \frac{\Gamma(5 q+1)}{\Gamma(4 q+1) \Gamma(q+1)}, \\
c_{3}^{6}=c_{1}^{5} c_{2}^{0}+c_{1}^{0} c_{2}^{5}+\left(c_{1}^{2} c_{2}^{3}+c_{1}^{3} c_{2}^{3}\right) \frac{\Gamma(5 q+1)}{\Gamma(3 q+1) \Gamma(2 q+1)}+\left(c_{1}^{1} c_{2}^{4}+c_{1}^{4} c_{2}^{1}\right) \frac{\Gamma(5 q+1)}{\Gamma(4 q+1) \Gamma(q+1)}-b c_{3}^{4} .
\end{array}\right.
\end{aligned}
$$


Thus, the solution of systems (5) is defined as

$$
\left\{\begin{array}{l}
x(t)=c_{1}^{0}+c_{1}^{1} \frac{h^{q}}{\Gamma(q+1)}+c_{1}^{2} \frac{h^{2 q}}{\Gamma(2 q+1)}+c_{1}^{3} \frac{h^{3 q}}{\Gamma(3 q+1)}+c_{1}^{4} \frac{h^{4 q}}{\Gamma(4 q+1)}+c_{1}^{5} \frac{h^{5 q}}{\Gamma(5 q+1)}+c_{1}^{6} \frac{h^{6 q}}{\Gamma(6 q+1)}, \\
y(t)=c_{2}^{0}+c_{2}^{1} \frac{h^{q}}{\Gamma(q+1)}+c_{2}^{2} \frac{h^{2 q}}{\Gamma(2 q+1)}+c_{2}^{3} \frac{h^{3 q}}{\Gamma(3 q+1)}+c_{2}^{4} \frac{h^{4 q}}{\Gamma(4 q+1)}+c_{2}^{5} \frac{h^{5 q}}{\Gamma(5 q+1)}+c_{2}^{6} \frac{h^{6 q}}{\Gamma(6 q+1)} \\
z(t)=c_{3}^{0}+c_{3}^{1} \frac{h^{q}}{\Gamma(q+1)}+c_{3}^{2} \frac{h^{2 q}}{\Gamma(2 q+1)}+c_{3}^{3} \frac{h^{3 q}}{\Gamma(3 q+1)}+c_{3}^{4} \frac{h^{4 q}}{\Gamma(4 q+1)}+c_{3}^{5} \frac{h^{5 q}}{\Gamma(5 q+1)}+c_{3}^{6} \frac{h^{6 q}}{\Gamma(6 q+1)}
\end{array}\right.
$$

When $a=5, b=2$, and $c=34$, system (5) has stable equilibrium with line $(0, h, 0)$. The analytical solution of the system can be obtained according to (14). Using MATLAB to simulate (14), the chaotic attractor is shown in Figure 4. The orbits marked by the blue and red colors emerge form initial $(1,2,3)$ and $(1,2,-1)$, and the red attractor is periodic. The blue attractors are shown in chaotic states.

\section{Dynamical Analysis}

Based on the ADM solution of the fractional-order chaotic system, we focus on the analysis of the influence of parameters on the system. In this paper, the system bifurcation diagram and Lyapunov exponent are used to analyze the dynamic system. The bifurcation diagram is obtained by using the maximum value of state variables. In this section, the influence of three parameters on the system is studied.

4.1. Dynamics with $z(0)$ Varying. Fix $a=5, b=2, c=34$, and $q=0.8$; initial values of state variables $\left[x_{0}, y_{0}, z_{0}\right]=\left[1,2, z_{0}\right]$; let the $z_{0}$ vary from -15 to 15 with step size of 0.01 . Bifurcation diagram and Lyapunov exponents spectrum of the system (5) are shown in Figure 5. As can be seen from the bifurcation diagram of the system in Figure 5(a), when $z_{0} \in[-15,-0.7]$, the system is in a periodic state and stable at $(0,0,0)$. When $z_{0} \in(-0.7,15]$, the system is in a chaos state. When $z_{0} \in[-15,-0.7]$, the largest Lyapunov exponents is equal to 0 . When $z_{0} \in(-0.7,15]$, the largest Lyapunov exponents is positive, as shown in Figure 5(b).

4.2. Dynamics with $q$ Varying. Fix $a=5, b=2$, and $c=34$; let the derivative order $q$ vary from 0.6 to 1 with step size of 0.002 and the initial values of state variables $\left[x_{0}, y_{0}, z_{0}\right]=[1$, $2,3]$. It can be seen from Figure 6(a) that, under the variation of derivative order $q$, the system chaos and periodic variation are repeated and crossed and are not a process from period to chaos directly. At the same time, Figure 6 shows that the system is chaotic over the interval $q \in[0.678,0.824) \cap[0.876,1]$. However, $q \in[0.876,1]$ also has a periodic window. It can be seen clearly that the maximum Lyapunov exponent decreases with the increase of derivative order $q \in[0.678,0.824]$. In this case, the minimum order for chaos is $q=0.678$. The maximum Lyapunov exponent value at this point $(q=0.678)$ is the largest. With the change of parameter $q$, the bifurcation diagram of the system is consistent with the Lyapunov exponent spectrum of the system.

For further research, influence of coexistence of attractors in fractional derivative $q$ system. In this paper, the phase diagram is used to introduce. It is found that $a=5, b=2$, and $c=34$. The integer-order system $(q=1)$ is different from the fractional-order system $(q=0.98)$. The attractors of the integerorder system are almost the same, while the attractors of the fractional-order system coexist. As shown in Figure 7, the red initial values are $[1,2,3]$. The blue initial values are $[1,2,20]$.

4.3. Dynamics with $a$ and $b$ Varying. The fractional chaotic system (5) has three system parameters besides the fractional derivative $q$. In this paper, the dynamic properties of the system parameters with a and $b$ varying are analyzed. Firstly, bifurcation diagrams of the fractional-order system with $a \in$ $[3,10]$ is investigated as shown in Figure $8(a)$, where the step size of $a$ is $0.05, q=0.8, b=2$, and the initial conditional is $\left[x_{0}, y_{0}, z_{0}\right]=[1,2,3]$. Obviously, it can be seen that $a \in[3.3,4.1] \cup[4.3,4.7] \cup[8.4,8.5]$ is in a periodic state; phase diagrams of the systems with different $a$ values are shown in Figure 9. Other regions are in chaotic state with periodic windows.

Secondly, bifurcation diagrams of the fractional-order system (5) with $b \in[0,6]$ is investigated as shown in Figure $8(\mathrm{~b})$, where the step size of $b$ is $0.05, a=5$, and the initial conditional is $\left[x_{0}, y_{0}, z_{0}\right]=[1,2,3]$. Obviously, it can be seen that $b \in[3.16,3.38] \cup[3.92,6]$ is in a periodic state, and other regions are in chaotic state with periodic windows.

QR decomposition method $[6,15]$ is effective in calculating the LEs, and its computational process is shown by

$$
\begin{aligned}
\operatorname{qr}\left[\mathbf{J}_{m} \mathbf{J}_{m-1}, \ldots, \mathbf{J}_{2} \mathbf{J}_{1}\right]= & \operatorname{qr}\left[\mathbf{J}_{m} \mathbf{J}_{m-1}, \ldots, \mathbf{J}_{2}\left(\mathbf{J}_{1} \mathbf{Q}_{0}\right)\right] \\
= & \operatorname{qr}\left[\mathbf{J}_{m} \mathbf{J}_{m-1}, \ldots, \mathbf{J}_{3}\left(\mathbf{J}_{2} \mathbf{Q}_{1}\right)\right] \times\left[\mathbf{R}_{1}\right] \\
= & \operatorname{qr}\left[\mathbf{J}_{m} \mathbf{J}_{m-1}, \ldots, \mathbf{J}_{4}\left(\mathbf{J}_{3} \mathbf{Q}_{2}\right)\right] \times\left[\mathbf{R}_{2} \mathbf{R}_{1}\right] \\
= & \ldots \\
= & \operatorname{qr}\left[\mathbf{J}_{m} \mathbf{J}_{m-1}, \ldots, \mathbf{J}_{i+1}\left(\mathbf{J}_{i} \mathbf{Q}_{i-1}\right)\right] \\
& \times\left[\mathbf{R}_{i-1} \mathbf{R}_{i-2}, \ldots, \mathbf{R}_{1}\right] \\
= & \mathbf{Q}_{m}\left[\mathbf{R}_{m} \mathbf{R}_{m-1}, \ldots, \mathbf{R}_{1}\right]=\mathbf{Q}_{m} \mathbf{R} .
\end{aligned}
$$

Here, $\mathrm{qr}($ ) represents QR decomposition function, $m$ is the maximum iteration number, and $J$ is the Jacobian matrix of 


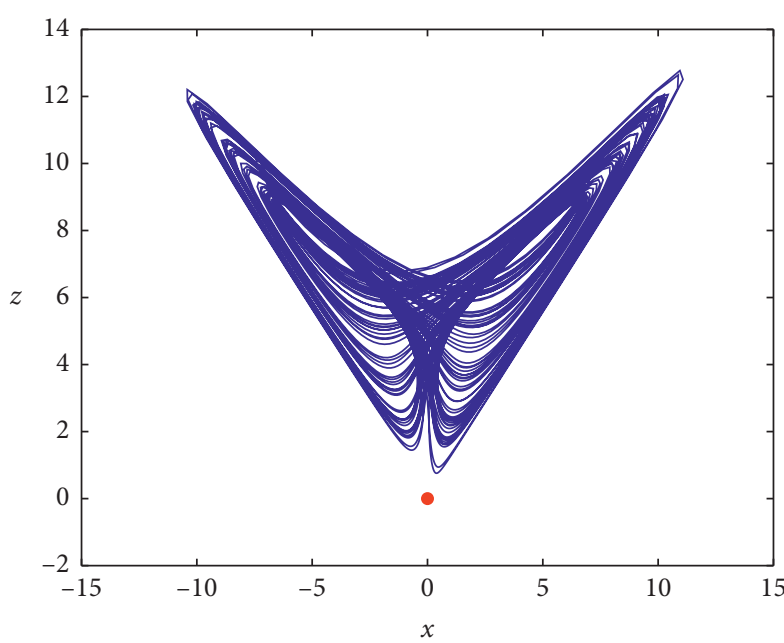

(a)

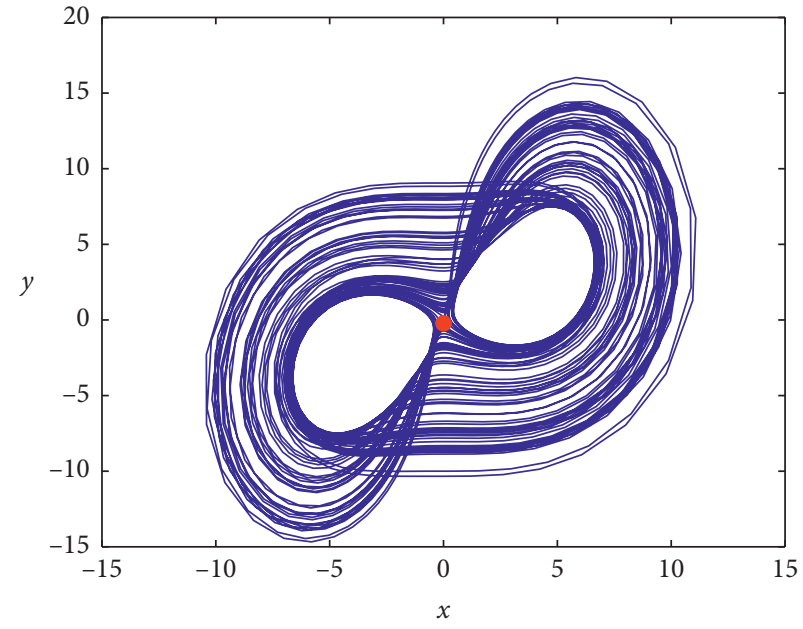

(b)

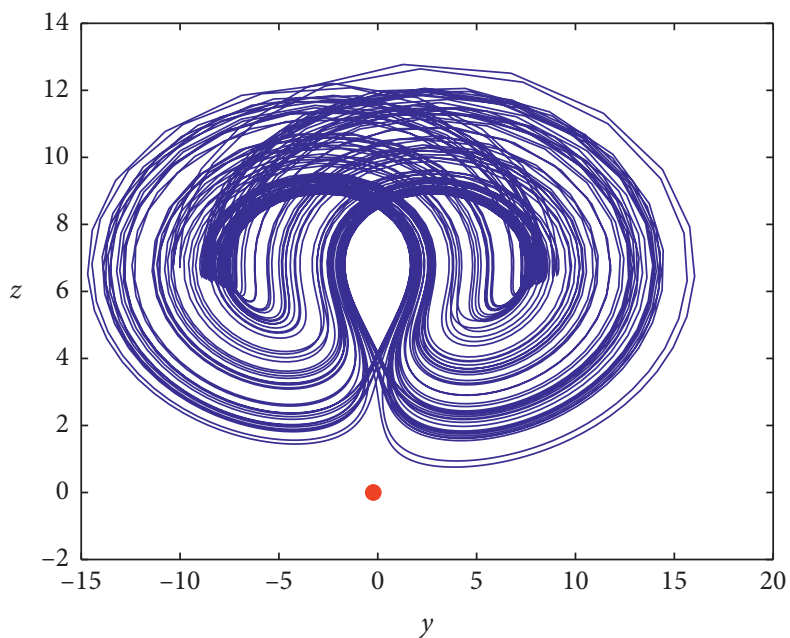

(c)

Figure 4: Phase diagrams in different projections of fractional-order chaotic systems from initial values $(1,2,3)$, red, and $(1,2,-1)$. (a) Phase portraits in the $(x)-(z)$ plane. (b) Phase portraits in the $(x)-(y)$ plane. (c) Phase portraits in the $(y)-(z)$ plane.

the given discrete formula as presented in (14). LCEs are calculated by

$$
\lambda_{k}=\frac{1}{\mathrm{mh}} \ln \left|\mathbf{R}_{i}(k, k)\right| \text {. }
$$

Here, $k=1,2, n$ (system dimension); $n$ is the maximum number of iterations; and $h$ is the iteration time step. According to the above basic formula, make $n=4, n=7000$, and $h=0.01$, and use MATLAB to draw the Lyapunov exponent under parameter changes.

Fix $b=2, q=0.8$; initial conditional is $\left[x_{0}, y_{0}, z_{0}\right]=[1,2,3]$; and let $a$ vary. Lyapunov exponents spectrum of system (5) is shown in Figure 10(a), which is consistent with the bifurcation diagram; namely, $a \in[3.3,4.1] \cup[4.3,4.7] \cup[8.4,8.5]$; the largest Lyapunov exponent of the system is equal to 0 (not positive), and the maximum Lyapunov exponent in other regions is greater than 0 (positive).

Fix $a=5, q=0.8$; initial conditional is $\left[x_{0}, y_{0}, z_{0}\right]=[1,2,3]$; and let $b$ vary. Lyapunov exponents spectrum of system (5) is shown in Figure 10(b), which is consistent with the bifurcation diagram; namely, $b \in[3.16,3.38] \cup[3.92,6]$; the largest Lyapunov exponent of the system is less than 0 (the phase diagram is a collection point), and the maximum Lyapunov exponent in other regions is greater than 0 (positive).

\section{Complexity Analysis of the Fractional- Order System}

5.1. Completeness Calculation. By calculating the energy distribution in the Fourier transform domain and combining it with the Shannon entropy, spectral entropy is obtained [31]. C0 complexity [32] is mainly to decompose the time series into regular and irregular parts and to test the proportion of irregular parts in the sequence. In practical applications, the chaos diagram of SE and C0 complexity can provide a basis to select parameter better.

Sample entropy (SampEn) [16] measures the complexity of time series by measuring the probability of generating new patterns in signals. The greater the probability of generation, the greater the value of the complexity of the sequence. 


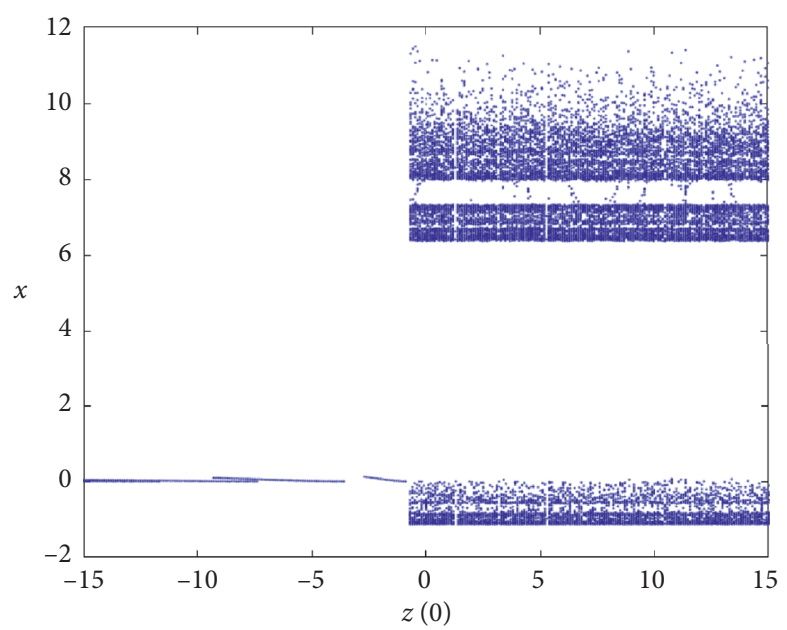

(a)

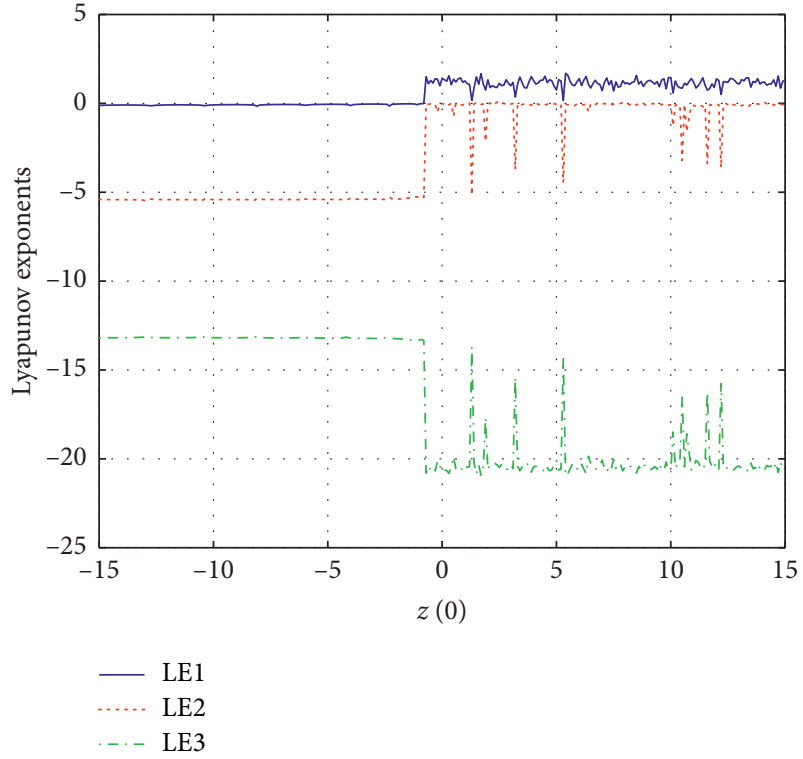

(b)

FIgURE 5: Bifurcation diagram and Lyapunov exponents spectrum of the fractional-order chaotic system (5) with initial conditional $\left[x_{0}, y_{0}\right.$, $\left.z_{0}\right]=\left[1,2, z_{0}\right]$. (a) Bifurcation diagram. (b) Lyapunov exponents (LEs).

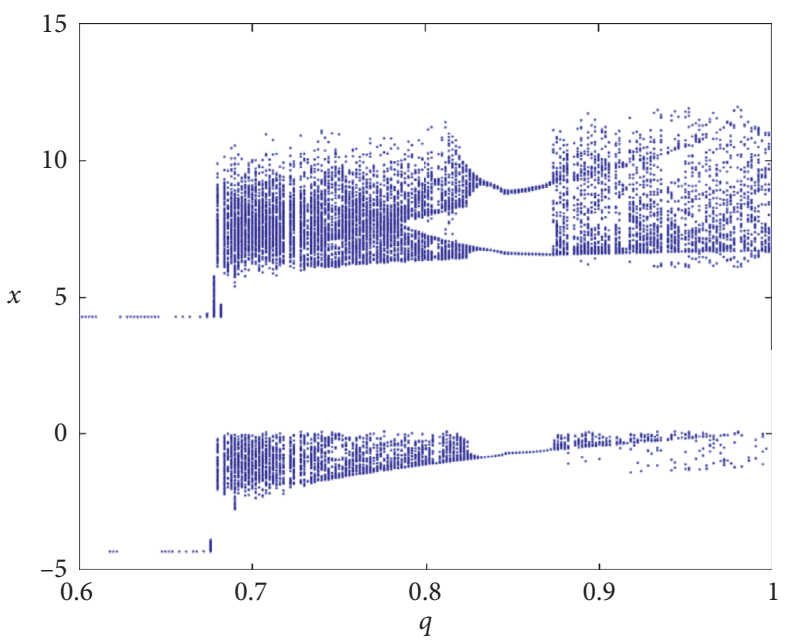

(a)

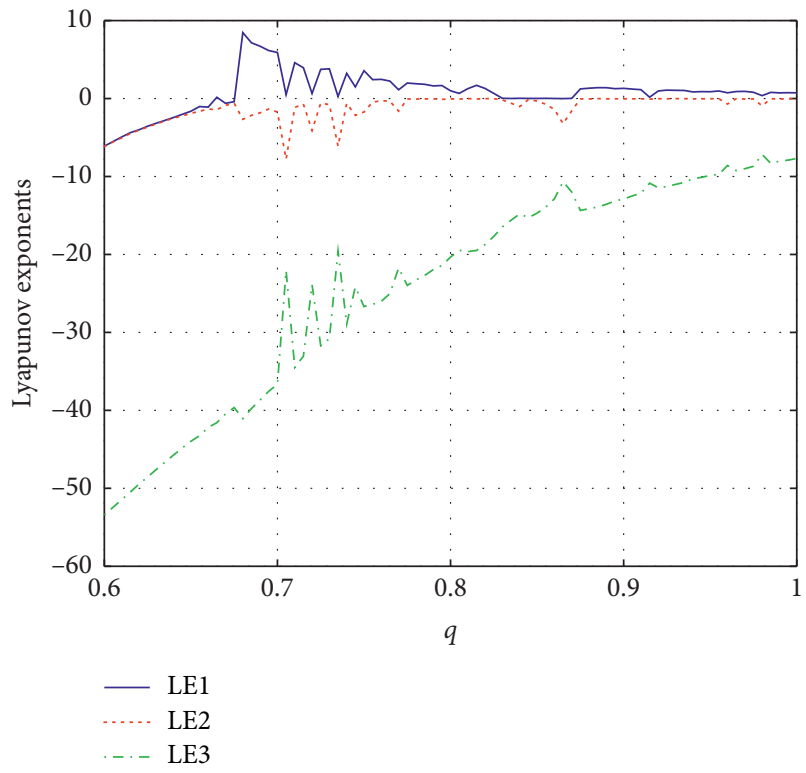

(b)

FiguRE 6: Bifurcation diagram and Lyapunov exponents spectrum of system (11) with $a=5, b=2$, and $c=34$. (a) Bifurcation diagram. (b) Lyapunov exponents (LEs).

In general, for the specific calculation method of sample entropy of time series $\{x(n)\}$ composed of $N$ data, the specific steps are as follows:

(i) S1: according to the serial number to form a onedimensional $m$ vector sequence, $\left\{X_{m}(1), \ldots\right.$, $\left.X_{m}(N-m+1)\right\}$, where $X_{m}(1)=\{x(i), x(i+1)$, $\ldots, x(i+m-1)\}, 1 \leq i \leq N-m+1$. These vectors are $m$ consecutive $x$ values starting from point 1 . (ii) S2: define the distance between $X_{m}(i)$ and $X_{m}(j)$ as $d\left[X_{m}(i) X_{m}(j)\right]$, as the difference between the maximum absolute values of the corresponding elements.

(iii) S3: define the criterion of similarity $r$. Count the number of $d\left[X_{m}(i), X_{m}(j)\right]<r(1 \leq j \leq N-m$, $1 \leq i \leq N-m)$, and its ratio to total distance $N-m$ is denoted as $B_{i}^{m}(r)=B_{i} /(N-m-1)$. 


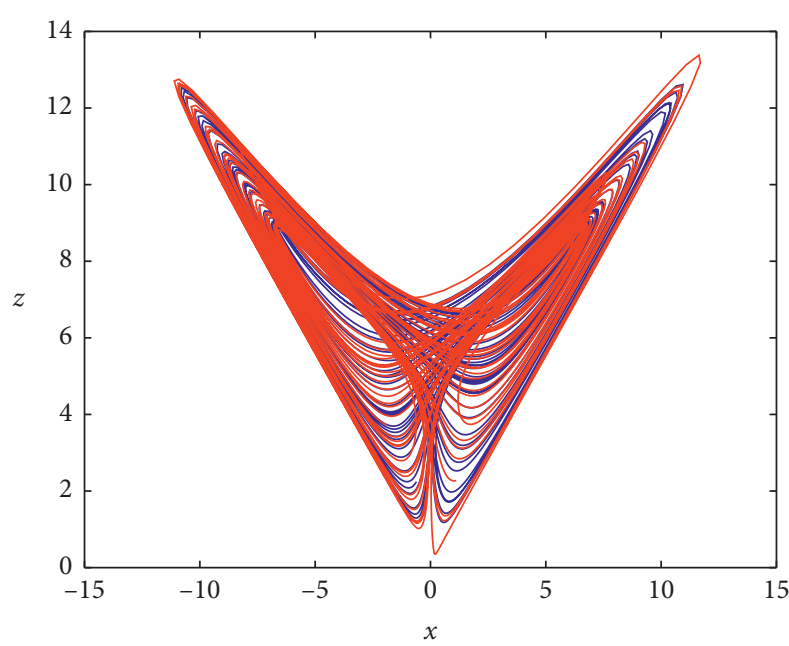

(a)

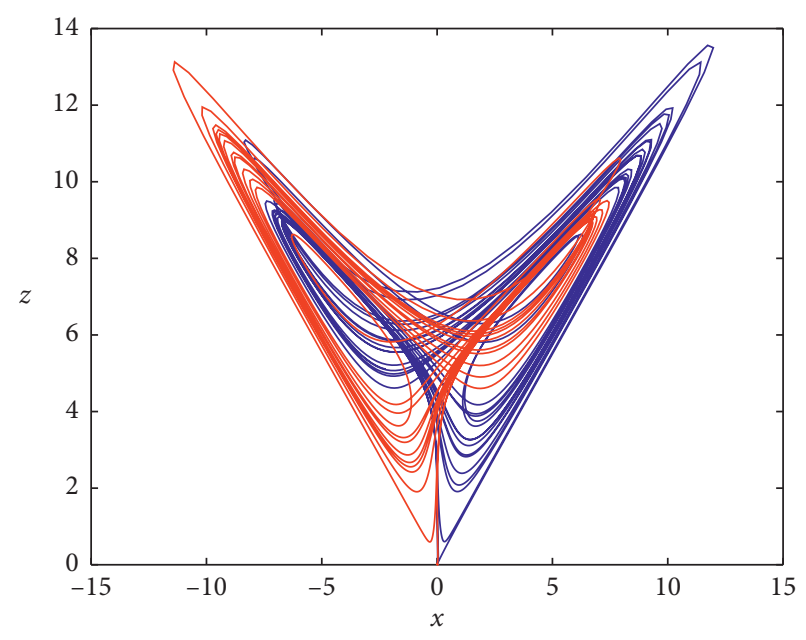

(b)

Figure 7: Phase diagrams in different projections of chaotic systems from initial values $(1,2,20)$ and $(1,2,3)$. (a) Phase diagrams in integerorder system (2). (b) Phase diagrams in fractional-order system (5).

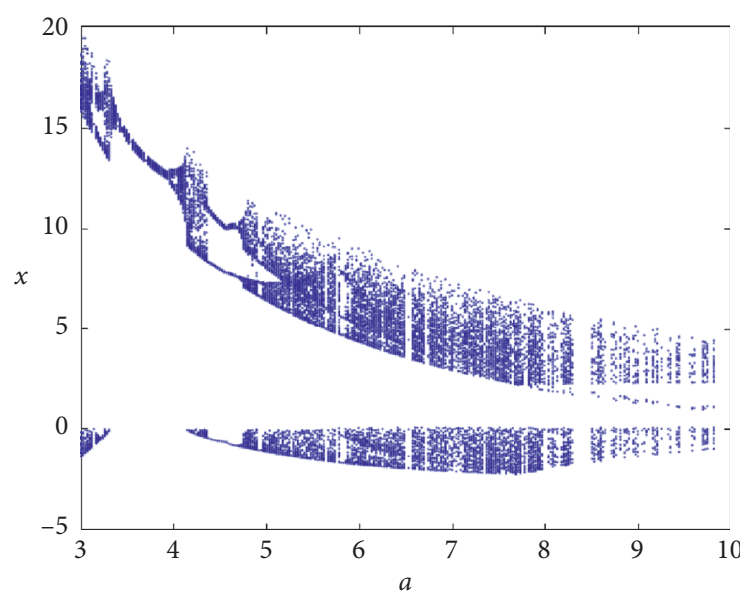

(a)

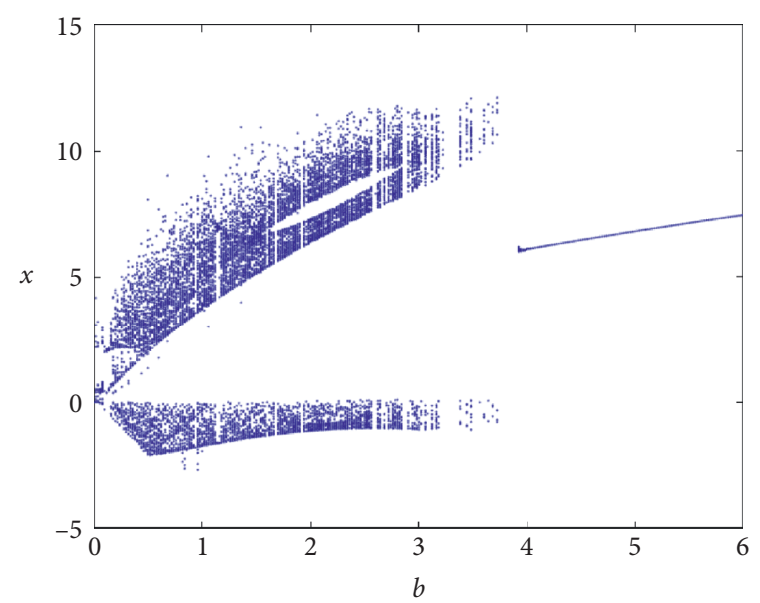

(b)

FIgURE 8: Dynamical properties of the fractional-order system with $a$ and $b$ varying. (a) $a$ varying. (b) $b$ varying.

(iv) S4: the average value of $B_{i}^{m}(r)$ can be calculated as

$$
B^{m}(r)=\frac{1}{N-m} \sum_{i=1}^{N-m} B_{i}^{m}(r)
$$

(v) S5: similarly, change $m$ to $m+1$ and repeat step one with step three; get $A^{m}(r)$.

(vi) S6: theoretically, the SampEn complexity can be calculated by

$$
\operatorname{SampEn}(m, r)=\lim _{N \longrightarrow \infty}\left\{-\ln \frac{A^{m}(r)}{B^{m}(r)}\right\} .
$$

5.2. Complexity Analysis. Fix $a=5, b=2, c=34$; let $q$ change from 0.6 to 1 ; step size is 0.002 ; and the initial value of state variables $\left[x_{0}, y_{0}, z_{0}\right]=[1,2,3]$. The complexity of the system is shown in Figure 11(a). We can see that the complexity decreases with the increase of $q$, and the change of complexity is consistent with the change of Lyapunov exponent in Figure 6(b).

Fixed $b=2, q=0.8$; initial conditional is $\left[x_{0}, y_{0}, z_{0}\right]=[1,2$, 3 ]; and let $a$ vary. The complexity of the system is shown in Figure 11(b). The change of complexity is consistent with the change of Lyapunov exponent in Figure 10(a).

Fix $a=2, q=0.8$; initial conditional is $\left[x_{0}, y_{0}, z_{0}\right]=[1,2$, $3]$; and let $b$ vary. The complexity of the system is shown in 


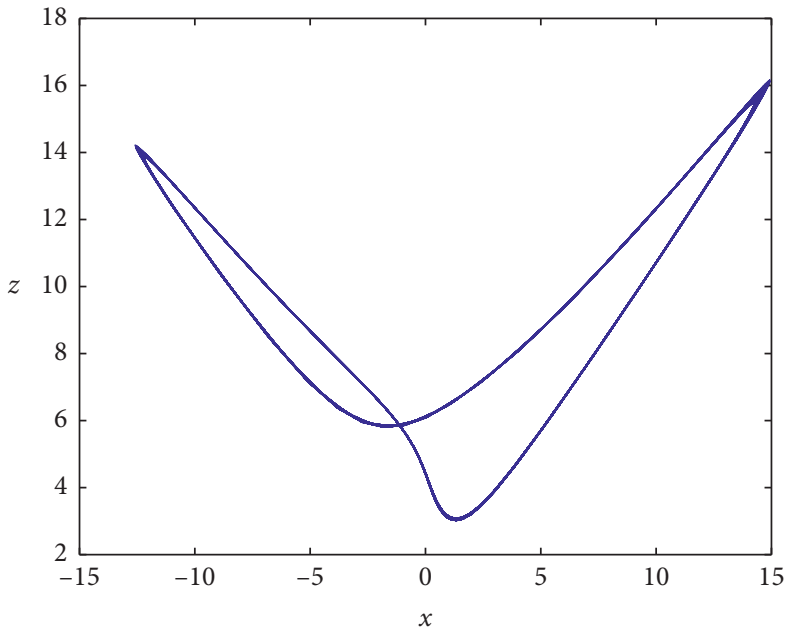

(a)

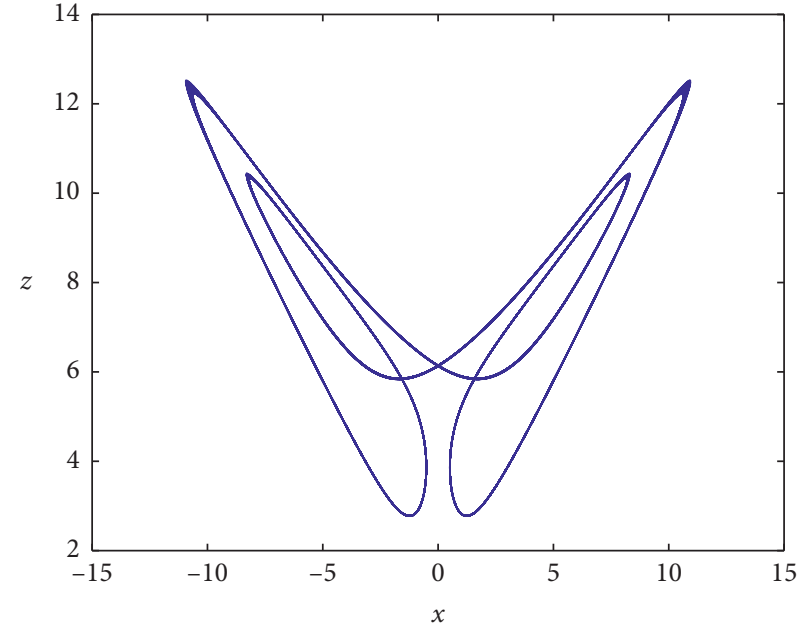

(b)

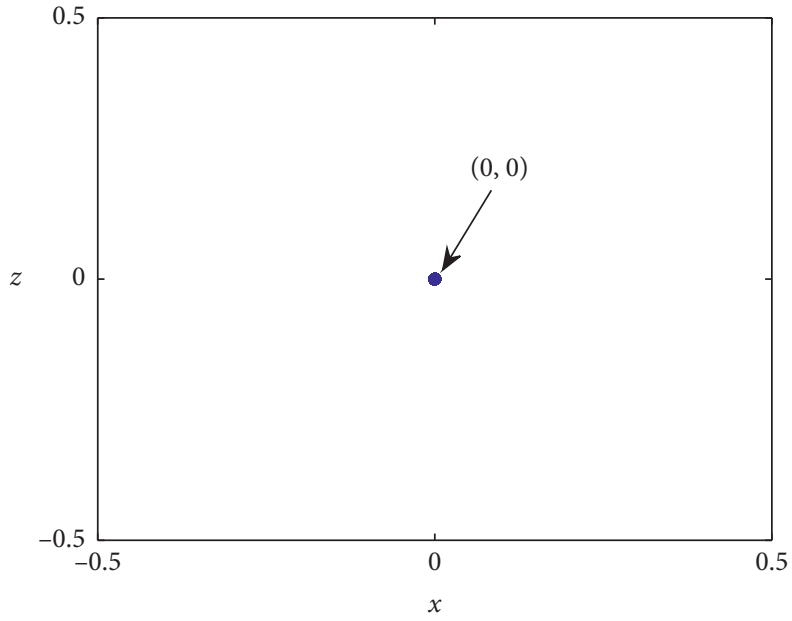

(c)

FIgURE 9: Phase diagrams of the systems with different $a$ values. (a) $a=3.5$. (b) $a=4.4$. (c) $a=8.46$.

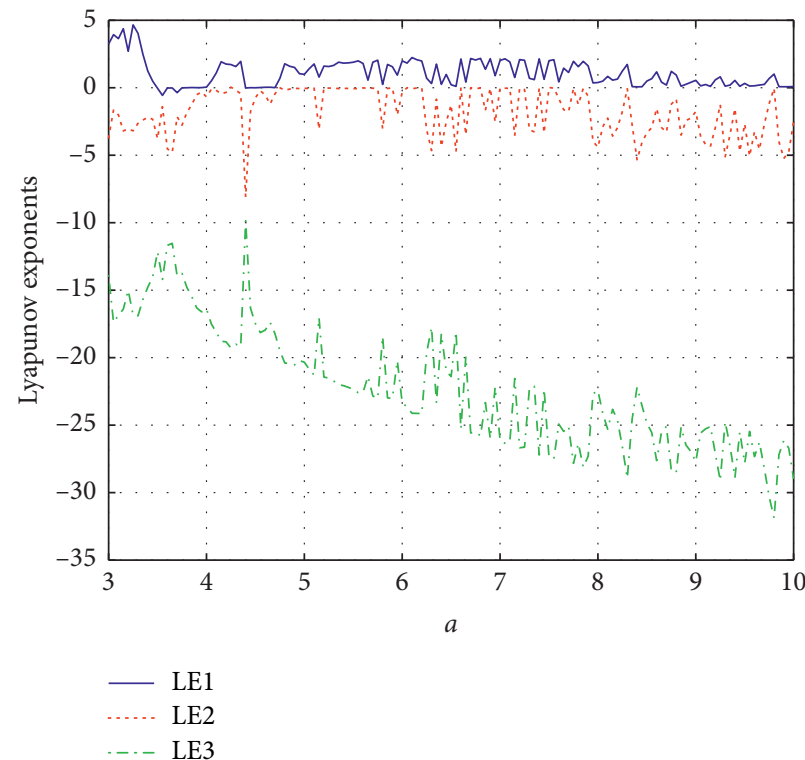

(a)

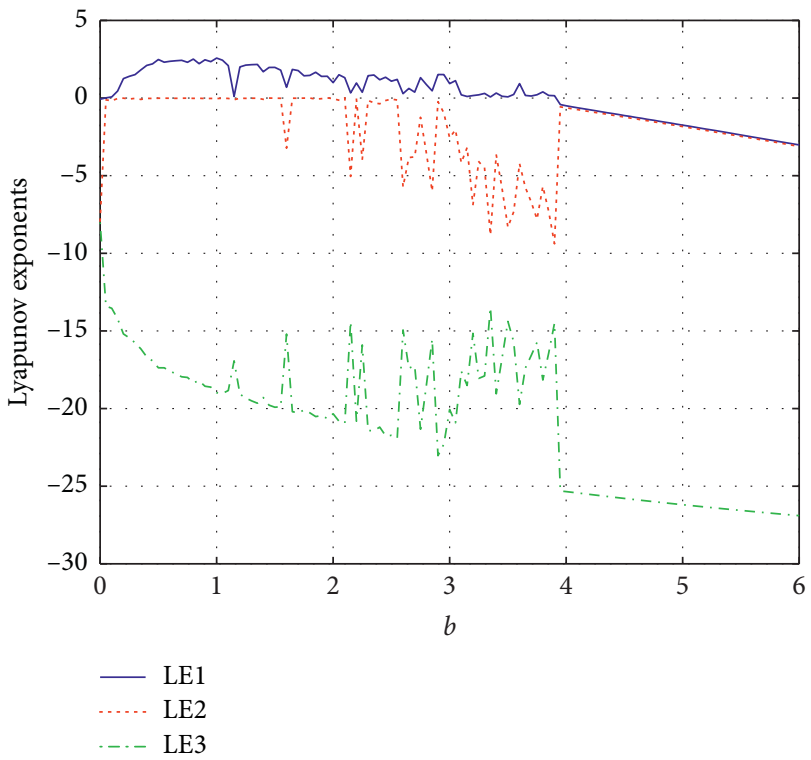

(b)

FIGURE 10: Lyapunov exponents spectrum of fractional order with $a$ and $b$ varying. (a) $a$ varying. (b) $b$ varying. 


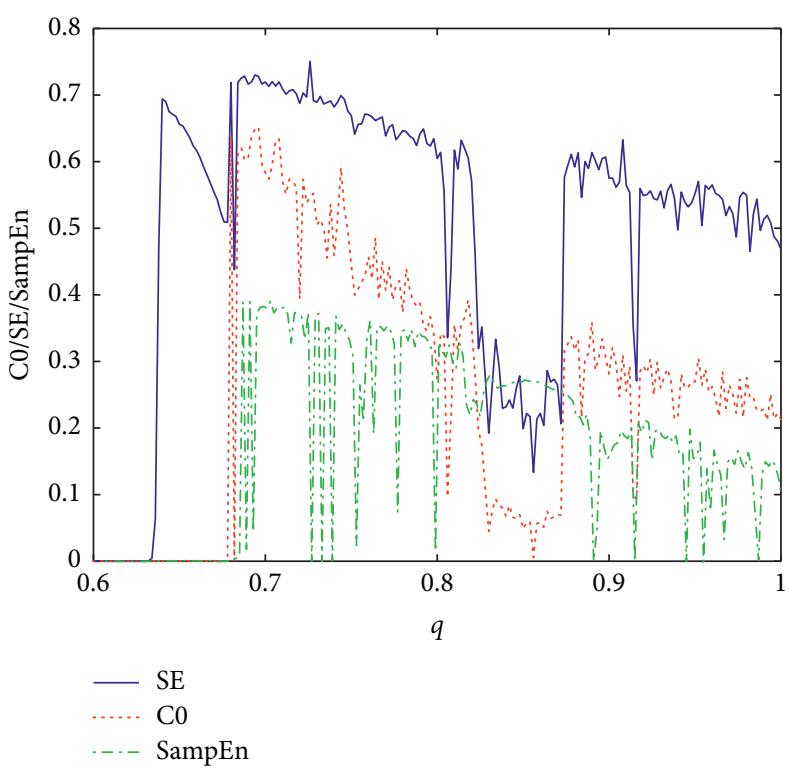

(a)

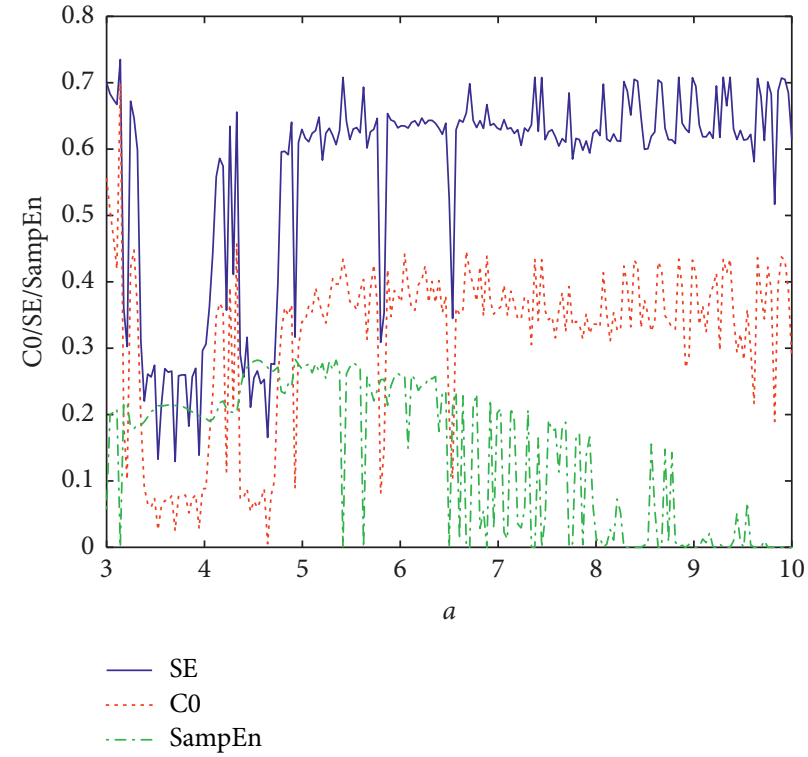

(b)

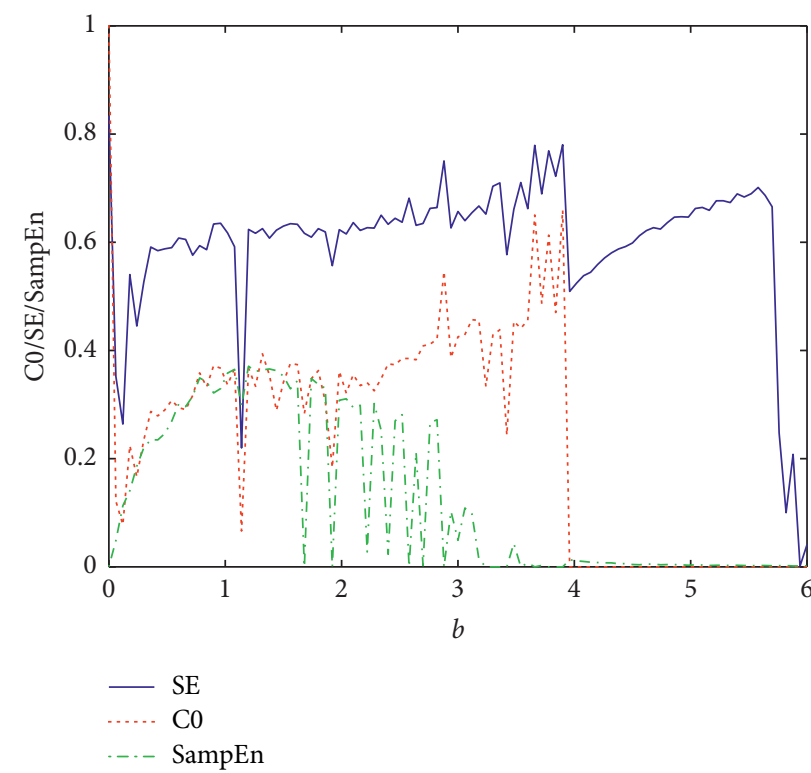

(c)

FIGURE 11: Complexity of the system with parameter varying. (a) $q$ varying. (b) $a$ varying. (c) $b$ varying.

Figure 11(c). The change of complexity is consistent with the change of Lyapunov exponent in Figure 10(b).

Complexity is consistent with Lyapunov exponent, but Figure 11 also shows that sample entropy complexity is small; SE complexity is not obvious when some bifurcation points change; $C_{0}$ complexity is accurate; and $C_{0}$ complexity is faster than Lyapunov exponent, consumes less resources, and provides a good idea for analysis of chaotic systems.

Fix $a=5, b=2$, and $c=34$; initial values of state variables $\left[x_{0}, y_{0}, z_{0}\right]=[1,2, z 0]$; let the $z(0)$ vary from 0 to 25 with step size of 0.2 and $q$ vary from 0.6 to 1 with step size of 0.004 . The C0 complexity in the $z(0)-q$ plane is shown in Figure 12. It can be seen that, in the fractional-order region, the system complexity under the change of $z(0)$ is more complex than that of the integer order.

The complexity of the system is calculated by the time sequence of a certain state variable. Compared with Lyapunov exponent calculation, the calculation of complexity is faster and saves resources, but there are also some errors in complexity, especially when SE complexity is in a periodic state. 


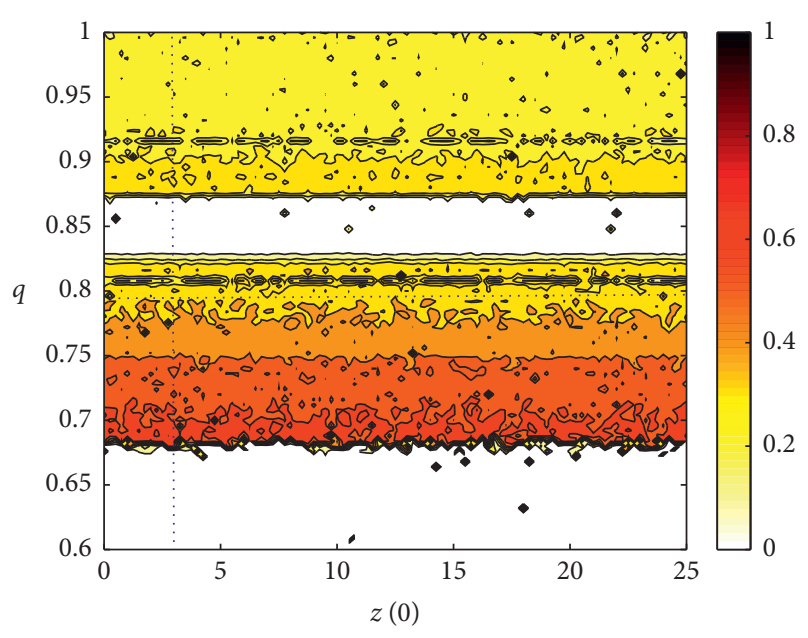

FIGURE 12: $\mathrm{C}_{0}$ complexity in the $z(0)-q$ plane.

\section{Conclusion}

In this paper, the accurate approximate solution for the fractional-order system with line equilibrium is obtained based on the Adomian decomposition method. Dynamical behaviors of the systems are analyzed using the chaotic attractor, bifurcation diagram, Lyapunov exponent spectrum, and largest Lyapunov exponent diagram. Chaotic range and periodic windows are determined. Both the system parameter and the fractional order can be taken as bifurcation parameters, which shows that the fractionalorder system has more complex and abundant dynamics than its integral-order counterpart. Besides, integer-order systems do not have the phenomenon of attractors coexistence, while fractional-order systems have it, so fractionalorder systems have better effects when applied to communication security systems.

\section{Data Availability}

The data used to support the findings of this study are available from the corresponding author upon request.

\section{Conflicts of Interest}

The authors declare that they have no conflicts of interest.

\section{Acknowledgments}

This work was supported financially by the Key Research and Development Plan of Shaanxi Province (No. 2018GY-091), the Special Fund for High Level Talents of Xijing University (XJ19B03), the Major Scientific and Technological Innovation Projects of Shandong Province (Grant No. 2019JZZY010111), the Natural Science Foundation of Shandong Province (Grant No. ZR2017PA008), the Key Research and Development Plan of Shandong Province (Grant No. 2019GGX104092), and the Science and Technology Plan Projects of Universities of Shandong Province (Grant No. J18KA381).

\section{References}

[1] K. I. Bolotin, F. Ghahari, M. D. Shulman, H. L. Stormer, and P. Kim, "Observation of the fractional quantum hall effect in graphene,” Nature, vol. 462, no. 7270, p. 196, 2009.

[2] V. E. Tarasov and G. M. Zaslavsky, "Fractional dynamics of coupled oscillators with long-range interaction," Chaos: An Interdisciplinary Journal of Nonlinear Science, vol. 16, no. 2, Article ID 023110, 580 pages, 2006.

[3] O. P. Agrawal, "A general formulation and solution scheme for fractional optimal control problems," Nonlinear Dynamics, vol. 38, no. 1-4, pp. 323-337, 2004.

[4] P. J. Torvik and R. L. Bagley, "On the appearance of the fractional derivative in the behavior of real materials," Journal of Applied Mechanics, vol. 51, no. 2, pp. 725-728, 1984.

[5] Y. Wang, K. Sun, S. He, and H. Wang, "Dynamics of fractional-order sinusoidally forced simplified Lorenz system and its synchronization," The European Physical Journal Special Topics, vol. 223, no. 8, pp. 1591-1600, 2014.

[6] S. He, K. Sun, and H. Wang, "Complexity analysis and DSP implementation of the fractional-order Lorenz hyperchaotic system," Entropy, vol. 17, no. 12, pp. 8299-8311, 2015.

[7] H. Jia, Z. Guo, G. Qi, and Z. Chen, "Analysis of a fourwing fractional-order chaotic system via frequency-domain and time-domain approaches and circuit implementation for secure communication," Optik, vol. 155, pp. 233-241, 2018.

[8] S. He, K. Sun, H. Wang, X. Mei, and Y. Sun, "Generalized synchronization of fractional-order hyperchaotic systems and its DSP implementation," Nonlinear Dynamics, vol. 92, no. 1, pp. 85-96, 2018.

[9] C. Li, K. Su, Y. Tong, and H. Li, "Robust synchronization for a class of fractional-order chaotic and hyperchaotic systems," Optik - International Journal for Light and Electron Optics, vol. 124, no. 18, pp. 3242-3245, 2013.

[10] S. B. He, S. Banerjee, and B. Yan, "Chaos and symbol complexity in a conformable fractional-order memcapacitor system," Complexity, vol. 2018, Article ID 4140762, 15 pages, 2018.

[11] A. Charef, H. H. Sun, Y. Y. Tsao, and B. Onaral, "Fractal system as represented by singularity function," IEEE Transactions on Automatic Control, vol. 37, no. 9, pp. 1465-1470, 1992.

[12] G. Adomian, "A review of the decomposition method and some recent results for nonlinear equations," Mathematical and Computer Modelling, vol. 13, no. 7, pp. 17-43, 1990.

[13] W. Deng, "Short memory principle and a predictor-corrector approach for fractional differential equations," Journal of Computational and Applied Mathematics, vol. 206, no. 1, pp. 174-188, 2007.

[14] K. Diethelm, N. J. Ford, and A. D. Freed, "A predictor-corrector approach for the numerical solution of fractional differential equations," Nonlinear Dynamics, vol. 29, no. 1-4, pp. 3-22, 2002.

[15] M. S. Tavazoei and M. Haeri, "Unreliability of frequencydomain approximation in recognising chaos in fractionalorder systems," IET Signal Processing, vol. 1, no. 4, pp. 171-181, 2007.

[16] H. Shao-Bo, S. Ke-Hui, and W. Hui-Hai, "Solution of the fractional-order chaotic system based on Adomian decomposition algorithm and its complexity analysis," Acta Physica Sinica, vol. 63, no. 3, Article ID 030502, 8 pages, 2014.

[17] B. Yan and S. He, "Dynamics and complexity analysis of the conformable fractional-order two-machine interconnected 
power system," Mathematical Methods in the Applied Sciences, vol. 7270, pp. 1-16, 2019.

[18] S. Zhou and X. Wang, "Identifying the linear region based on machine learning to calculate the largest Lyapunov exponent from chaotic time series," Chaos: An Interdisciplinary Journal of Nonlinear Science, vol. 28, no. 12, Article ID 123118, 2018.

[19] S. Zhou, X. Wang, Z. Wang, and C. Zhang, "A novel method based on the pseudo-orbits to calculate the largest Lyapunov exponent from chaotic equations," Chaos: An Interdisciplinary Journal of Nonlinear Science, vol. 29, no. 3, Article ID 033125, 2019.

[20] C. Li, T. Lu, G. Chen, and H. Xing, "Doubling the coexisting attractors," Chaos: An Interdisciplinary Journal of Nonlinear Science, vol. 29, no. 5, Article ID 051102, 2019.

[21] C. Li, Y. Xu, G. Chen, Y. Liu, and J. Zheng, "Conditional symmetry: Bond for attractor growing," Nonlinear Dynamics, vol. 95, no. 2, pp. 1245-1256, 2018.

[22] X. Wang and G. Chen, "A chaotic system with only one stable equilibrium," Communications in Nonlinear Science and Numerical Simulation, vol. 17, no. 3, pp. 1264-1272, 2012.

[23] S. Jafari, J. C. Sprott, and M. Molaie, "A simple chaotic flow with a plane of equilibria," International Journal of Bifurcation and Chaos, vol. 26, no. 6, Article ID 1650098, 2016.

[24] S. Jafari, A. Ahmadi, A. J. M. Khalaf, H. Reza Abdolmohammadi, V.-T. Pham, and F. E. Alsaadi, "A new hidden chaotic attractor with extreme multi-stability," AEU International Journal of Electronics \& Communications, vol. 89, pp. 131-135, 2018.

[25] B. Hamdi and S. Hassen, "A new hypersensitive hyperchaotic system with No equilibria," International Journal of Bifurcation and Chaos, vol. 27, no. 5, Article ID 1750064, 2017.

[26] X. Y. Hu, C. X. Liu, L. Liu, Y. P. Yao, and G. C. Zheng, "Multiscroll hidden attractors and multi-wing hidden attractors in a 5-dimensional memristive system," Chinese Physics B, vol. 26, no. 11, 130 pages, Article ID 110502, 2017.

[27] K. E. Chlouverakis and J. C. Sprott, "Chaotic hyperjerk systems," Chaos, Solitons \& Fractals, vol. 28, no. 3, pp. 739-746, 2006.

[28] C. Li, J. C. Sprott, and W. Thio, Linearization of the Lorenz systemPhysics Letters A, vol. 379, no. 10-11, pp. 888-889, 2015.

[29] Z. Wang, "Existence of attractor and control of a 3D differential system," Nonlinear Dynamics, vol. 60, no. 3, pp. 369-373, 2009.

[30] G. Adomian, "A new approach to nonlinear partial differential equations," Journal of Mathematical Analysis and Applications, vol. 102, no. 2, pp. 420-434, 1984.

[31] P. A. Phillip, F. L. Chiu, and S. J. Nick, "Rapidly detecting disorder in rhythmic biological signals: a spectral entropy measure to identify cardiac arrhythmias," Physical Review E, vol. 79, no. 1, Article ID 011915, 2009.

[32] E. H. Shen, Z. J. Cai, and F. J. Gu, "Mathematical foundation of a new complexity measure," Applied Mathematics \& Mechanics, vol. 26, no. 9, pp. 1188-1196, 2005. 